\title{
Inhaltsverzeichnis
}

\section{Versicherungs-Statistik für 1906}

Finloitung . . . . . . . . . . . . . . . . $7^{*}$ Allgemeiner Ülıorblick. . . . . . . . . . . . . . . $\mathrm{S}^{*}$ Gruppe I: Lebensversicherung und Krankonversicherung . . . . . . . . . . . $16^{*}$

Gruppe 11: Unfall- und Haftpflichtvorsicherung . . 39*

Gruppo III: Vieh- und Hagelversicherung . . . . 48*

Gruppe IV: Feuervorsicherung sowie Versicherung gegen Sturmschäden, Wasserleitungsschäden and EinbruchdiebstahI . . . .

Gruppe V: Glasversicherung, Kautions- und Garantiovorsichorung, Kredit-, Maschinen-, Kaskovorsicherung usw. . . . . . . . $71^{*}$

Tafel.

\section{Tabe11en \\ Gruppe I}

Vorzeichnis der Abkürzungen für dio Namen der Uuteruchmungen . . . . . . . . . . .

\section{A. Versicherungsbestand Ende 1906 Deutsche allgemeine Unternehmungen$$
\text { GroBo Versichorang }
$$

Tab. 1 1: Gesamtbestand der überhanpt übernommenen, der in Rückdeckung gegebenen Versicherungen und der Versicherungen für eigeno Rechnung. . . . . . . . . . . .

" I 2: Dio überhaupt übernommenen Versicherungen nach Versicherungsarten . . . . . . .

"I 3: Dio in Rückdeckung gegebenen Vorsicherungen und die Versicherungen für eigeno Rechnung nach Versicherungsarten . . .

"I 4: Bestand der selbst abgeschlossenen und der in Rückdockung übornommenen Versicherungen auf den Todesfall, zerlegt nach dor Gewinnbeteiligung . . . . . . . . .

"I 5: Bestand dor selbst abgeschlossenen und der in Rückdeckung übernommenen Versicherungen auf den Lebensfall, zerlegt nach der Gewinnbeteiligung . . . . . . . . .

"I 6: Bestand der selbst abgeschlossenen und der in Rückdeckung übernommenen Rentenversicherungen, zerlegt nach der Gewinnbeteiligung

Seito
T'al. I 7: Bestand der selbst alugeschlossenen und der in Rückdeckung übernommenen Invaliditätsversicherungen, zerlegt nach der Gewinnbeteiligung . . . ... . . . . . .

"I 8: Bestand der Krankenversichorung, zcrlegt nach der Gewinnbeteiligung . . . . . .

\section{Kloine Vorsicherung}

"I9: Bestand der überhaupt ubbernommenon, dor in Rückdeckung gegebenen Versicherungen und der Versicherungen für eigene Rechnung

"I 10: Die selbst abgeschlossenen und dio in Rückdockung übernommenen Versicherungen auf den Todosfall, zerlegt nach der Gewinnbeteiligung . . . . . . . . . . . .

"I 11: Die selbst abgeschlossenon und die in Rückdecknng übernommenen Versicherungen anf den Lobensfall, zerlegt nach der Gowinnbeteiligung . . . . . . . . . . .

Versicherungseinrichtangen größerer deutseher Berufsvereinigungen

"I 12: Die Vorsicherungen, zerlogt nach dor Gewinnbeteilignng

\section{Auslinndische Gesellschaften}

GroBo Vursichorung - Doutschor Bestand

'Tab.I 13: Bestand der überhaupt übernommenen, der in Rückdeckung gegebenen Versichorungen und der Versicherungen für eigeno Rechnung

"114: Dio überhaupt äbernommenen Versichorungen nach Versicherungsarten - Verhältnis des doutschen Geschäfts zum ganzen Goschäfte . . . . . . . . . . . . . 38

"11j: Dio in Rückdeckung gegebenen Versicherungen und die Versicherungon für oigene Rochnung nach Versicherungsarten. . . .

"116: Bestand dor selbst abgeschlossenen und der in Rückdeckung übernommenen Versichorungen auf den Todesfall, zerlegt nach der Gewinnbeteiligung . . . . . . . . .

"I 17: Bestand der Versicherungen auf den Lebensfall, zerlegt nach der Gowinnbeteiligung .

"I18: Bestand dor Rentenversicherungen, zerlegt nach der Gewrinnbeteiligung - Invaliditätsversicherung . . . . . . . . . . 45 
Kloino Versicherung - Dentscher Bestand

T'ab. 1 19: Bestand der überhaupt übernommenen, der in Rückdeckung gegebenou Versicherungen und der Versicherungen für oigene Rechnung - Verhältnis dos deutschen Goschäfts zum ganzen Geschäfte . . . . . . . . . .

" 1 20: Bestand der Versicherangen, zerlegt nach der Gewinnbeteiligung . . . . . . . .

\section{B. Bewegung im Versicherungsbestand im Jahre 1906}

\section{Deutsche allgemeine Unternehmungen}

$$
\text { Grobe Versicherung }
$$

"J 21: Bewegung in der Versicherung auf den Todesfall . . . . . . . . . . .

"I 22: Bewegung in der Versichcrung auf den Lebensfall . . . . . . . . . . .

" I 23: Bowegung in der Rentenversicherung. . .

" I 24: Bowegung in dor Invaliditätsversicherung .

" 125 : Bowegung in der Krankenversicherung . .

Kloine Versicherung

"126: Bewegung in der Versicherung auf den T'odesfall . . . . . . . . . . . .

"I 27: Bewegung in der Versicherung auf don Lebensfall . . . . . . . . . . .

- Versicherungseinrichtungen größerer deutscher Berufsrereinigungen

"I 28: Bowegung in der Versicherung . . . . .

\section{Ausländische Gesellschaften}

GroBe Versichorung - Deutscher Bestand " 129: Bewegung in der Versicherung auf den Todesfall . . . . . . . . . . . . . "I 30: Bewegung in der Versicherung auf den Lebensfall . . . . . . . . . . .

"I 31 : Bewegung in dor Rentenversicherung . .

Kleine Versichorung - Deutscher Bestand

"132: Bewegung in der Versicherung auf den Todesfall und auf den Lebensfall . . . . 96

\section{Betriebsrechnung für 1906}

\section{Veutsche allgemeine Unternehmungen}

$$
\text { GroBe Versicherung }
$$

"I 33: Gewinn- und Vorlustrechnung . . . . .

" I 34: Die Einnahmen aus Prämien und die Vergütnng der Rückversicherer sowie die Ausgaben für Versicherungsfalle des Geschäftsjahrs und für Rückversicherung : . . . . 10

„1 35 : Das finanzielle Ergebnis aus der Sterblichkeit
Kleine Vorsicherung

I'ab. 136: Gewinn- und Verlustrochnung . . . . . 116

"I 37: Die Einnahmen aus Prämien und die Vergütung der Rückvorsicherer sowio dio Ausgaben für Vorsicherungsfällo dos Geschäftsjahrs und für Rückvorsichorung.

\section{Versicherungseinrichtangen größerer} deutseher Berufsvereinigungen

"I 38: Gewinn- und Verlustrechnung . . . . .

"1 139: Die Einnahımen aus Prämien und dio Vergütung dor Rückrersicheror sowie die Ausgaben für Versicherungsfälle dos Gesehäftsjahrs und für Rückversicherung . . . . 128

\section{Ausländische Gesellschaften}

GroBe und kleine Versichorung " 140: Gewinn- und Verlustrochnung für das Gesamtgeschäft . . . . . . . . . . .

"141: Deutsches Geschäft, die Einnahmen aus Prämien und dio Vorgütung der Rückversicheror sowie die Ausgaben für Versicherungsfällo des Geschäftsjahrs und für Rïckversicherung. . . . . . . . . . . . 135

D. Bilanz für den SehluB des Jahres 1906 Deutsche allgemeine Unternehmungen

" I 42: Bilanz . . . . . . . . . . . . . . 138

"1 43: Die Art der Kapitalanlagen und der Aktiv forderungen . . . . . . . . . . . . . 146

" I 44: Die Prämienreserven und die Prämienüborträgo nach Versichorungsarten . . 152

„I 45: Dio Vorteilung des Gowinns . . . . . . 156

"I 46: Die Art und der Betrag der Ende 1906 vorhandenen Wertpapiere (Nennwert) . . . . 160

" 147: Die Bewegung der Gesellschaftsfonds in Jahre 1906. . . . . . . . . . . . 164

Vorsicherangseinrichtangen größerer deutscher Berufsvereinigungen

"I 48: Bilanz . . . . . . . . . . . . . . 168

" 149: Die Art der Kapitalanlagen und der Aktivforderungen . . . . . . . . . . 172

„150: Die Verteilung des Gewinns . . . . . 174

"I 51 : Die Art und der Betrag der Ende 1906 vorhandenen Wertpapiere (Nennwert). . . . 176

" 152: Die Bewegung der Gesellschaftsfonds im Jahre 1906 . . . . . . . . . 178

Ausländische Gesellschaften

" I 53: Bilanz . . . . . . . . . . . . . 180

"I 54: Die Art der Kapitalanlagon und der Aktivforderungen . . . . . . . . . 184

\section{E. Prämienreservefonds}

"I 55: Dentsche allgemeine Unternehmungen . . 186

"I 56: Versicherangseinrichtungen größerer dentscher Berufsvereinigungen . . . . 190

" I 57: Ausländische Unternehmungen . . . . 192 


\section{Gruppe II}

Verzeichuis dor Abkürzungen für dio Namen dor Unternehunungen . . . . . . . . . . . 195

\section{Deutsche Unternehmungen}

T'ab II 1: Die Zahl der Ende 1906 laufenden Vorsicherungen und die Prämien des Goschäftsjahrs

" II 2: Vergleich des Geschäftsjahrs mit dem Vorjahro

,

II 3: Die Betriebs-Einnahmon und Ausgaben und der Betriebsgewinn der Unfall- und Haftpflichtversicherung .

II 4: Dio Zahlungen für selbst abgeschlossone Versicherungen nnd die Vergütungen für in Rückdeckung übernommene Versicherungen

, 11 5: Die Betriebs-Einnahmen und Ausgaben aller Versicherungszweige . . . . . . . . 2116

„II 6: Gewinn- und Verlustrechnung . . . . 208

"II 7: Bilanz . . . . . . . . . . . . . 212

"II 8: Die Art der Kapitalanlagen und der Aktivforderungen . . . . . . . . . . . 214

„Il 9: Dio Verteilung des Gewinns . . . . . 214

" II 10: Die Art and der Betrag der Endo 1906 vorhandenen Wertpapiero (Nonnwert) . . . 216

" I111: Die Bewegung der Gesellschaftsfonds in Jahre 1906 . . . . . . . . . 216

"II 12: Die Eintragungen in das Prämienresorveregister. . . . . . . . . . . . 218

\section{Ausländische Gesellschaften}

Deutsches Geschäft

„ Il 13: Dio Zahl der Ende 1906 laufenden Versicherungen und die Prämien des Geschäftsjahrs . . . . . . . . . . . . .

" I1 14: Vergloich des Geschäftsjahrs mit doin Vorjahre . . . . . . . . . . . . . 220

" 1115: Die Zahlungen für sellbst abgeschlossene Versicherungen und dio Vergütungen für in Rückdeckung übernommene Versicherungen

\section{Gesamtgeschäft}

" 11 16: Die Betriebs-Einnahmen und Ausgaben der Unfall- und Haftpflichtversicherung . . . 224

„ 1I17: Gewinn- und Verlustrechnung . . . . 226

" Il 18: Bilanz . . . . . . . . . . . . . 228

" II 19: Die Art der Kapitalanlagon und der Aktivforderungen . . . . . . . . . . . 224

" Il 20: Die Eintragungen in das Prämienreserveregister. . . . . . . . . . . . . 230

\section{Gruppe III}

Verzeichnis der Abkürzungen für die Namen der Unternehmungen .. . . . . . . . . . 23

\section{Viehversicherung}

Tab. III 1: Umfang der Viehversicherung im Geschäftsjahro 1906 . . . . . . . . . . . 233

" III 2: Zerlegung des Geschäfts nach Versicherungsarten
'Tab) III 3: Vergloich des Geschäftsjahrs mit dem Vorjahro. . . . . . . . . . . . . . 237

"III 4: Gewinn- und Verlustrechnung. . . . . 238

" III 5: Erläuterung dor in die Gewinn- und Ver lustrechnung eingestellten Prämien und Schäden . . . . . . . . . . . 242

„ III 6: Bilanz . . . . . . . . . . . . . 244

"III 7: Die Art der Kapitalanlagen und der Aktirforderungen . . . . . . . . . . . 248

" III 8: Dio Verteilung des Gewinns . . . . 254

"III 9: Dio Art und der Betrag der Endo 1906 vorhandenon Wertpapiere (Nennwert) . . . 250

"III 10: Dio Bervogung dor Gesellschaftsfonds in Jahre 1906 . . . . . . . . . . . 252

\section{Hagelversicherung}

" III 11: Umfang dor Hagelversicherung in Geschäftsjahro 1906 . . . . . . . . . 255

"III 12: Bewegung in dor Hagelversicherung . . 256

" III 13: Gewinn- und Verlustrechnung . . . . . $25 \mathrm{~S}$

" 111 14: Erläuterung dor in dio Gewinn- und Verlustrechnung eingestellten Prämien und Schäden . . . . . . . . . . . . 262

"III 15: Bilanz . . . . . . . . . . . . . 264

"III 16: Die Art der Kapitalanlagen und der Aktivforderungen . . . . . . . . . $26 \mathrm{~S}$

" III 17: Die Verteilung des Gowinns . . . . . 274

" III 18: Die Art und der Betrag der Ende 1906 vorhandenen Wertpapiere (Nennwert) . . . 270

" 111 19: Dio Bewegung der Gesellschaftsfonds im Jahro 1906 . . . . . . . . . . . 272

\section{Gruppe IV}

Verzeichnis der Abkürzungen für die Namen der Unteruchmungen

\section{Versicherangsbestand Ende 1905 und Ende 1906}

Doutscho Unternohmungen

T'ah. IV 1: Zahl und Betrag der Feuorversichorungen - Gesamtgeschäft . . . . . . . 278 u. 284

" IV 2: Zahl und Betrag der Feuerversicherungon - Inländisches Geschäft . . . . . . 280

„IV 3: Zahl und Betrag der Feuerversicherungen - Ausländisches Geschäft . . . . . . 282

"IV 4: Zahl und Betrag der Sturmschäden- und dor Wasserleitungsschädenversicherungen . . 285

"IV 5: Zahl und Betrag dor Einbruchdiebstahlversicherungen - Gesamtgeschäft . . . 288

"IV 6: Zahl und Betrag der Einbruchdiebstahlversicherungen - Inländisches Geschäft . 290

" IV 7: Zahl und Betrag der Einbruchdiebstahlversichorungen - Ausländisches Geschäft 292

\section{Ausländische Gesellschafton-} Doutschos Geschät

"IV 8: Zahl and Betrag der Fouerversichorungen 294

"IV 9: Zahl und Betrag der Einbruchdiebstahlversicherungen . . . . . . . . . . 294 
Prümien und Schäden im Jahre 1906

Doutsche Untornohmungen

T'ab. IV 10: Die Prämien und die Schäden aus der Feuerversicherung . . . . . . . . . 296

„IV 11: Die Prämien und dic Schäden aus der Sturmschäden- und der Wasserleitungsschädenversicherung . . . . . . . . 310

"IV 12: Die Prămien und dio Schäden aus der Einbruchdiobstahlversicherung. . . . . 312

Ausländische Gesellschaften Deutsches Geschät

" IV 13: Dio Prämicn und dic Schäden aus der Feucrversicherung . . . . . . . . . 204

" IV 14: Die Prämion und die Schäden aus der Einbruchdiebstahlversichorung. . . . 304

Betriebsrechnung für 1906

Doutscho Unternehmungon

„IV 15: Die Betricbs-Einnalmmen und Ausgaben und dor Betriebsgewinn der Feuerversicherung 306

" IV 16: Die Betriebs-Einnahmen und Ausgaben und der Betriebsgewinn der Sturmschäden- und der Wasserleitungsschädenversicherung .

" IV 17: Die Betriebs-Einnahmen und Ausgaben und der Betriebsgewinn der Einbruchdiobstahlversicherung . . . . . . . . . . . 312

"IV 18: Die Betriebs-Einnahmen und Ausgaben allor Versicherungszweige . . . . . . . . 314

" IV 19: Gewinn- und Verlustrechnung . . . . 318

Ausländische Gesellschaften Gesamtgeschäf

"IV 20: Dio Betriebs-Einnahmon und Ausgaben aus der Fenerversicherung . . . . . . .

"IV 21 : Dio Betriebs-Einnahmen und Ausgaben aus der Einbruchdiebstahlversicherung . . . 326

"IV 22: Die Betriobs-Einnahmon und Ausgaben aller Versicherungszweigo . . . . . . . . 328

"IV 23: Gowinn- und Verlustrechuung . . . . 330

\section{Bilanz fïr den Schlnß des Jahres 1906}

Doutsche Unternehmungen

"IV 24: Bilanz . . . . . . . . . . . . . 334

" IV 25: Die Art der Kapitalanlagen und der Aktivforderungen . . . . . . . . . . . 342

„IV 26: Die Verteilung des Gewinns . . . . . 346

" IV 27: Die Art und der Botrag der Ende 1906 rorhandenen Wertpapiere (Nennwert) . . . 350

" IV 28: Dio Bewegung der Gesellschaftsfonds ini Jahre 1906

Ausländische Gesellschaften

"IV 29: Bilanz . . . . . . . . . . . . . 358

" IV 30: Die Art der Kapitalanlagen und der Aktivforderungen

\section{Gruppe v}

Verzeichnis der Abkürzungen für dio Naneu der Untornehmungen . . . . . . . . . 365

\section{A. Glasversichernng}

Versicherungsbestand Ende 1905 and Ende 1906 Deutsche Unternehmungen

Tab. V 1: Zahl und Betrag der Glasversicherungen - Gesamtgeschäft. . . . . . . . . 368

" V 2: Zahl und Betrag der Glasversicherungen— Inländisches Geschäft . . . . . . 370

" V 3: Zahl and Betrag der Glasversicherungen - Ausländisches Geschäft . . . . . . 372

A usländischo GosellschaftonDeutsches Geschät

"V 4: Zahl und Betrag der Glasversicherungen . 374

\section{Prämien und Schäden im Jahre 1906}

„V 5: Die Prämien und die Schäden aus dem Glasversicherungsgeschäfte der doutschen Unternehmungon . . . . . . . . . . . 376

" V G: Die Prämien und die Schäden aus dem deutschen Glasversicherungsgeschäfte der ausländischen Gesellschaften . . . . 374

\section{Betriebsrechnnng für 1906}

Doutsche Gosollschafton

" V 7: Die Botriebs-Einnahmen und Ausgaben und der Botriobsgewinn der Glasvorsicherung 378

" V S: Gewinn- und Vorlustrechnung . . . . 380

Bilanz fïr den SchluB des Jahres 1906

Deutsche Gesellschafiten

"V 9: Bilanz... . . . . . . . . . . . 384

"V 10: Die Art dor Kapitalanlagen und der Aktivforderungen . . . . . . . . . . . 388

"V 11: Die Verteilung des Gewinns . . . . . 388

"V 12: Die Art und der Betrag der Ende 1906 vorhandenen Wertpapiere (Nennwert) . . . 390

V 13: Die Bewegung der Gesollschaftsfonds in Jahre 1906 . . . . . . . . . . 390

B. Kantions-, Garantie-, Kredit-, Maschinen-, FahrzeugUnfall-, Baulast- und Wertgegenstände-Versicherung

\section{Doutscho Gesellschafton}

V 14: Zahl und Botrag der Versichorungen . 392

V 15: Die Prämien und die Schäden . . . . 396

V 16: Die Betricbs-Einnahmon und Ausgaben und der Betriebsgewinn . . . . . . . 398

\section{Kaskoversicherung}

Deutsche Unternohmungen

V 17: Zahl und Betrag der Kaskoversicherungen 406

V 18: Gewinn- und Verlustrechnung . . . . 400

V 19: Vermögensübersicht . . . . . . . . 404

Berichtigungen . . . . . . . . . 407 